\title{
Causas de defunción mal definidas en las provincias de Argentina, 2001-2013
}

\author{
Bruno Sebastián Ribotta
}

Centro de Investigaciones y Estudios sobre Cultura y Sociedad (CIECS). Consejo Nacional de Investigaciones Científicas y Técnicas (CONICET). Universidad Nacional de Córdoba (UNC), Argentina.

Cómo referenciar este artículo/
How to reference this article:
Ribotta B. Causas de defunción mal definidas en las provincias de Argentina, 2001-2013. Mem. Inst. Investig. Cienc. Salud. 2016;14(3):86-95

\section{RE S U M E N}

El objetivo fue analizar los niveles y tendencias en el porcentaje de muertes con causas mal definidas en las provincias de Argentina, durante los años 2001 a 2013. Este fue un estudio descriptivo, de tipo cuantitativo. Se utilizó la base de datos de registros de defunción de la Dirección de Estadísticas e Información en Salud. A partir del porcentaje de defunciones con causas mal definidas, se clasificó a las provincias según niveles de exactitud: alta $(<5 \%)$, buena $(\geq 5 \%<10 \%)$, regular $(\geq 10 \%<20 \%)$ y baja $(\geq 20 \%)$. Se analizó la distribución relativa de las muertes según el grupo de códigos más frecuentes: signos y síntomas (R00-R69), hallazgos anormales clínicos y de laboratorio (R70-R94) y causas desconocidas y mal definidas propiamente dichas (R95-R99). La tendencia temporal se estableció mediante el cambio porcentual. En 11 provincias la exactitud fue alta, en 5 buena y en 8 regular. En todas las jurisdicciones, los códigos más frecuentes fueron las causas desconocidas o mal definidas propiamente dichas, aunque éstas fueron proporcionalmente menos importantes en las provincias con nivel alto de exactitud. Además, en este grupo de jurisdicciones se observaron las reducciones más importantes del porcentaje de causas mal definidas. Solo dos provincias con niveles intermedios de exactitud presentaron la misma pauta. El análisis de las causas de muerte mal definidas, permitió reconocer problemas básicos en la información, y proporcionó indicios válidos para investigaciones futuras. Resulta de interés identificar las buenas prácticas implementadas en las provincias menos afectadas por este inconveniente.

Palabras clave: Estadísticas vitales, registros de defunción, causa básica de muerte, causas mal definidas, calidad de los datos, Argentina.

\section{Ill-defined causes of death in the provinces of Argentina, 2001-2013}

\section{A B S T R A C T}

The objective was to analyze levels and trends in the percentage of deaths with ill-defined causes in the provinces of Argentina, during the years 2001-2013. This was a quantitative descriptive study. Database of death records from the Department of Health Statistics and Information was used. From the percentage of deaths with ill-defined causes, provinces were classified into four accuracy levels: high $(<5 \%)$, good $(\geq 5 \%<10 \%)$, regular $(\geq 10 \%$ $<20 \%$ ) and low ( $\geq 20 \%$ ). The relative distribution of deaths was analyzed according to the group of more frequent codes: symptoms and signs (R00-R69), abnormal clinical and laboratory findings (R70-R94) and ill-defined and unknown causes of death (R95-R99). Time trend was established with the relative variation. Eleven provinces had an excellent level of accuracy, five provinces had a very good level of accuracy and eight provinces had a good level of accuracy. In all jurisdictions, the most common codes were the unknown or ill-defined causes, although they were proportionally less important in the provinces with high accuracy. Besides, the most significant reductions were observed in this group. Only 
two provinces with intermediate levels showed the same pattern. The analysis of the illdefined causes of death identified the most basic problems in the information, and provided valid evidence to guide future research. It is interesting to analyze the good practices that have been implemented by the provinces that are less affected by this problem.

Key Words: Vital Statistics, death records, cause of death, ill-defined cause of death, data quality, Argentina.

\section{INTRODUCCIÓN}

La causa básica de muerte corresponde a la enfermedad o lesión que inicia la cadena de acontecimientos patológicos que llevan directamente a la muerte de una persona, o las circunstancias del accidente o violencia que dan lugar a una lesión fatal (1).

La recolección, sistematización y difusión de la información sobre la causa básica de muerte a través de sistemas de registro civil y estadísticas vitales, constituye una tarea de importancia para los países, en la medida en que contribuye a la evaluación y el seguimiento del estado de salud de la población, y a la planificación de intervenciones sanitarias. De la misma forma, cuando se registran de manera oportuna, los datos sobre la causa básica de muerte pueden utilizarse para la determinación temprana de cambios en la prevalencia de ciertas enfermedades, facilitando la elaboración de programas de prevención o de intervención (2). Especial mención merece el uso de esta información para el seguimiento de alianzas nacionales e internacionales realizadas para el mejoramiento de los resultados en salud, como la Conferencia Sanitaria Panamericana y los Objetivos de Desarrollo del Milenio (ODM), entre otros. Algunos ejemplos son la razón de mortalidad materna, y las tasas de mortalidad por VIH/Sida o por tuberculosis.

La información sobre la causa básica muerte se obtiene a través de un complejo proceso de producción, que generalmente difiere de un país a otro al involucrar a diferentes instituciones (establecimientos de salud, Registro Civil, Oficina Nacional de Estadísticas), etapas (diseño del instrumento de recolección, relevamiento y procesamiento de los datos, difusión y uso), y responsables (médicos, registradores civiles, informáticos y estadísticos), que deben ser regulados por un marco teórico y técnico muy específico (3). Al mismo tiempo, se requiere de una nomenclatura especial, con claves preestablecidas para posibilitar la identificación de las causas de muerte, su almacenamiento y agregación. Actualmente, este requisito debe cumplirse con la $10^{\circ}$ Revisión de la Clasificación Internacional de Enfermedades (CIE-10). Dicha clasificación garantiza las comparaciones internacionales, y se actualiza y revisa frecuentemente, para contemplar los conocimientos más avanzados sobre las causas de los principales trastornos de salud y enfermedades (4).

En este contexto, existen una serie de factores que pueden dar lugar a errores en la información sobre la causa básica de muerte. Por ejemplo, cuando el médico certificante desconoce todas las causas intervinientes en la defunción, y por ello determina los motivos del deceso de manera incompleta o poco exhaustiva. En otras situaciones, cuando los profesionales evitan dar detalles sobre los motivos que dieron lugar a la muerte, ya que suponen equivocadamente que la información puede ser utilizada en procesos judiciales (5). Otras motivaciones de la inexactitud en los datos resultan de la escasa capacitación en el empleo de la CIE-10, en la falta de claridad en el instrumento de recolección de datos (certificado y/o informe estadístico de defunción), y en la existencia de muertes informadas por agentes no médicos (generalmente, las que ocurren fuera de los establecimientos de salud) (6).

Para estudiar los errores en la información sobre la causa básica de muerte, uno de los enfoques más corrientes radica en la consideración de las "causas mal definidas". Esta denominación corresponde a los signos, síntomas y hallazgos clínicos y de laboratorio no clasificados en otra parte de la CIE-10. Dichas causas se identifican mediante los códigos R00-R99, que comprenden las situaciones en que el médico o la persona que realiza la certificación de la muerte, no logra diagnosticar la causa básica de defunción (7).

El porcentaje de defunciones con causas mal definidas (PCMD), constituye un indicador utilizado corrientemente para evaluar la exactitud de los datos. Como regla general, cuanto menor sea el resultado obtenido mayor será la precisión de la información recolectada. Por el contrario, cuando dicha proporción resulta elevada la distribución de las causas de muerte aparece sesgada y es poco confiable (8). Al respecto, los umbrales pueden ser más o menos concretos. Algunos autores sostienen que los datos son excelentes cuando el porcentaje de causas mal definidas no supera al $5 \%$ en menores de 65 años, ni al $10 \%$ en mayores de esa 
edad. Otros aclaran que la exactitud es óptima cuando dicho porcentaje no supera al $10 \%$, intermedia cuando oscila entre $10 \%$ y $20 \%$, y baja cuando supera al $20 \%(8-10)$.

Actualmente, de 119 países miembros de OMS que registran estadísticamente a las defunciones, solo 34 poseen datos de elevada exactitud sobre la causa básica de muerte (9). En América Latina, se han realizado esfuerzos muy importantes para mejorar los sistemas de información sobre mortalidad. Entre los avances más destacados, se cuenta que todos los países que producen datos de este tipo utilizan la CIE-10 para certificar las causas de muerte, y que la gran mayoría cuenta con menos de $10 \%$ de las defunciones mal definidas (11).

En Argentina, el porcentaje de muertes con causas mal definidas se mantiene por debajo del $10 \%$, aunque se ha incrementado en los últimos años $(12,13)$. Al respecto, el indicador ascendía a $6,1 \%$ en el año 2001, momento en que se implementa el nuevo Informe Estadístico de Defunción, y a $8,4 \%$ una década después. Esta circunstancia llama la atención en un país que se destaca por la elevada cobertura, oportunidad y disponibilidad de la información sobre mortalidad, y señala la importancia de implementar medidas que puedan revertir la dirección actual.

Dada la extensión territorial y la diversidad socio-cultural que presenta el país, interesa analizar la situación de las causas mal definidas en las divisiones político-administrativas de mayor tamaño que componen el país; las provincias y la Ciudad Autónoma de Buenos Aires (Capital Federal). El estudio de la exactitud de las estadísticas vitales a este nivel de desagregación, es importante ya que cada una de estas jurisdicciones, a través de su respectiva Oficina de estadísticas de salud, actúa entre la captación de los datos a nivel local (establecimientos de salud y registros civiles), y su remisión anual al nivel nacional (Ministerio de Salud), y allí realiza el primer control, codificación e informatización de los registros (14).

En este marco, el objetivo de este trabajo es evaluar la situación actual y de la tendencia reciente del porcentaje de causas mal definidas en la Capital Federal y las 23 provincias que componen la Argentina. De esta manera, se espera aportar evidencias que permitan formular recomendaciones para el fortalecimiento de la información sobre mortalidad del país y proponer líneas de investigación futura.

\section{MATERIALES Y MÉTODOS}

Se realiza un estudio cuantitativo, de tipo descriptivo. Ello implica el relevamiento y evaluación de datos secundarios (registros de defunción), la estimación de indicadores para la identificación de las pautas más importantes, y su análisis a partir del estado de conocimiento. El periodo en estudio se extiende desde la implementación del nuevo Informe Estadístico de Defunción de la Argentina (2001), al último año con información disponible (2013). A continuación se detalla la fuente de datos y los indicadores utilizados:

- Fuentes de información: Se utiliza una base de datos provista por la Dirección de Estadísticas e Información de Salud (DEIS) del Ministerio de Salud de la Nación, procesada con el software SPSS. La misma incluye las muertes registradas oficialmente en el país a partir del Subsistema de Estadísticas Vitales, con la causa básica codificada a cuatro caracteres de la $10^{\circ}$ Revisión de la Clasificación Internacional de Enfermedades (CIE-10).

- Indicadores: Se calcula la proporción de muertes con causas "mal definidas" respecto al total de defunciones, por 100. Asimismo, se clasifica a este tipo de defunciones en los siguientes grupos: signos y síntomas (códigos R00-R69), hallazgos clínicos y de laboratorio (códigos R70-R94) y causas desconocidas o mal definidas propiamente dichas (códigos R95R99). Luego se estima la proporción que representa cada uno de los tres conjuntos en el total de las muertes mal definidas, por 100.

En las estimaciones se utilizan promedios móviles de tres años, para evitar las fluctuaciones que pudieran resultar de cantidades reducidas de casos. Los resultados se muestran desagregados según lugar de residencia, considerando las 23 provincias y la Capital Federal (Ciudad Autónoma de Buenos Aires). Se distribuye proporcionalmente a las defunciones con datos ignorados en esta última variable.

Para el análisis del nivel de exactitud, se clasifica a cada provincia en cuatro grupos según el PCMD: 1)- excelente (menos de $5 \%), 2$ )- buena (5\% a $10 \%), 3)$ - regular $(10 \%$ a $20 \%$ ) y baja ( $20 \%$ o más). Esta clasificación constituye una adaptación de criterios corrientes para catalogar la calidad de los registros de defunción (8-10).

La tendencia temporal se establece mediante la variación relativa de los porcentajes. De manera accesoria, los cambios más importantes se indican con cifras absolutas. Para la 
caracterización del nivel se tiene en cuenta el trienio 2011-2013, y para la evaluación de la tendencia temporal los periodos 2001-2003 y 2011-2013 (Tabla 1).

Tabla 1. Argentina. Defunciones mal definidas y total de defunciones, según provincia. 2001 a 2003 y 2011 a 2013

\begin{tabular}{|c|c|c|c|c|c|c|c|c|c|c|c|c|}
\hline \multirow{3}{*}{ Jurisdicción } & \multicolumn{12}{|c|}{ Defunciones según año y tipo (con causas mal definidas, total) } \\
\hline & \multicolumn{2}{|l|}{2001} & \multicolumn{2}{|l|}{2002} & \multicolumn{2}{|l|}{2003} & \multicolumn{2}{|l|}{2011} & \multicolumn{2}{|l|}{2012} & \multicolumn{2}{|l|}{2013} \\
\hline & CMD & Total & CMD & Total & CMD & Total & CMD & Total & CMD & Total & CMD & Total \\
\hline Capital Federal & 1.095 & 33.898 & 1.214 & 34.054 & 1.367 & 35.293 & 362 & 32.298 & 304 & 31.560 & 358 & 32.133 \\
\hline Buenos Aires & 6.376 & 117.268 & 7.331 & 118.049 & 8.036 & 122.301 & 12.617 & 131.626 & 11.121 & 132.349 & 12.406 & 133.035 \\
\hline Catamarca & 107 & 1.811 & 123 & 1.943 & 143 & 1.965 & 131 & 2.088 & 127 & 2.260 & 188 & 2.384 \\
\hline Córdoba & 1.347 & 24.453 & 1.404 & 24.739 & 1.590 & 26.480 & 1.919 & 27.834 & 2.115 & 27.497 & 1.998 & 28.755 \\
\hline Corrientes & 689 & 6.156 & 704 & 6.070 & 824 & 6.149 & 909 & 6.395 & 986 & 6.750 & 989 & 6.880 \\
\hline Chaco & 543 & 6.113 & 645 & 6.643 & 626 & 6.356 & 968 & 6.863 & 1.015 & 7.128 & 906 & 7.250 \\
\hline Chubut & 142 & 2.348 & 152 & 2.503 & 147 & 2.379 & 61 & 2.869 & 45 & 3.033 & 59 & 3.085 \\
\hline Entre Ríos & 1.046 & 8.756 & 1.265 & 9.160 & 1.375 & 9.710 & 1.764 & 10.019 & 1.765 & 9.667 & 1.901 & 10.065 \\
\hline Formosa & 189 & 2.780 & 220 & 3.015 & 170 & 2.818 & 152 & 3.292 & 149 & 3.392 & 150 & 3.537 \\
\hline Jujuy & 397 & 3.389 & 427 & 3.525 & 402 & 3.477 & 496 & 4.004 & 506 & 4.132 & 547 & 4.231 \\
\hline La Pampa & 66 & 2.147 & 94 & 2.255 & 88 & 2.386 & 63 & 2.518 & 57 & 2.482 & 46 & 2.608 \\
\hline La Rioja & 110 & 1.601 & 107 & 1.764 & 155 & 1.738 & 252 & 2.039 & 260 & 2.059 & 178 & 2.083 \\
\hline Mendoza & 652 & 11.129 & 847 & 11.297 & 1.059 & 11.905 & 317 & 12.873 & 325 & 12.879 & 278 & 13.130 \\
\hline Misiones & 584 & 4.488 & 634 & 5.575 & 689 & 5.527 & 117 & 6.108 & 199 & 6.507 & 70 & 6.603 \\
\hline Neuquén & 91 & 2.075 & 69 & 2.115 & 65 & 2.229 & 67 & 2.822 & 51 & 2.884 & 73 & 2.966 \\
\hline Río Negro & 270 & 2.973 & 321 & 3.108 & 371 & 3.353 & 528 & 4.014 & 487 & 3.899 & 498 & 4.114 \\
\hline Salta & 415 & 5.698 & 525 & 6.017 & 448 & 6.114 & 79 & 6.967 & 371 & 7.229 & 346 & 7.509 \\
\hline San Juan & 66 & 4.151 & 90 & 4.472 & 89 & 4.530 & 41 & 4.739 & 69 & 4.548 & 83 & 4.802 \\
\hline San Luis & 111 & 2.223 & 99 & 2.345 & 75 & 2.587 & 5 & 2.849 & 6 & 2.831 & 6 & 3.046 \\
\hline Santa Cruz & 36 & 985 & 24 & 1.054 & 33 & 1.019 & 121 & 1.288 & 94 & 1.378 & 112 & 1.401 \\
\hline Santa Fe & 2.225 & 26.424 & 2.315 & 26.819 & 2.610 & 28.652 & 4.152 & 29.217 & 3.766 & 28.404 & 3.378 & 29.784 \\
\hline S. del Estero & 776 & 4.424 & 809 & 4.322 & 855 & 4.549 & 834 & 5.187 & 903 & 5.251 & 998 & 5.548 \\
\hline Tucumán & 1.343 & 8.298 & 1.537 & 8.615 & 1.689 & 9.043 & 617 & 9.448 & 482 & 10.079 & 411 & 9.964 \\
\hline T. del Fuego & 16 & 277 & 16 & 305 & 14 & 316 & 16 & 450 & 19 & 502 & 12 & 479 \\
\hline Otro país & 10 & 175 & 15 & 184 & 17 & 194 & 10 & 249 & 16 & 250 & 18 & 272 \\
\hline Ignorado & 170 & 1.901 & 123 & 1.242 & 129 & 994 & 82 & 1.003 & 31 & 589 & 35 & 533 \\
\hline Total & 18.872 & 285.941 & 1.110 & 291.190 & 23.066 & 302.064 & 26.680 & 319.059 & 25.269 & 319.539 & 26.044 & 326.197 \\
\hline
\end{tabular}

Fuente: Procesamientos especiales de bases de datos de la DEIS, Argentina.

\section{RESULTADOS}

La Figura 1 presenta el PCMD registrado en Argentina a nivel nacional y provincial durante el trienio 2011-2013. 


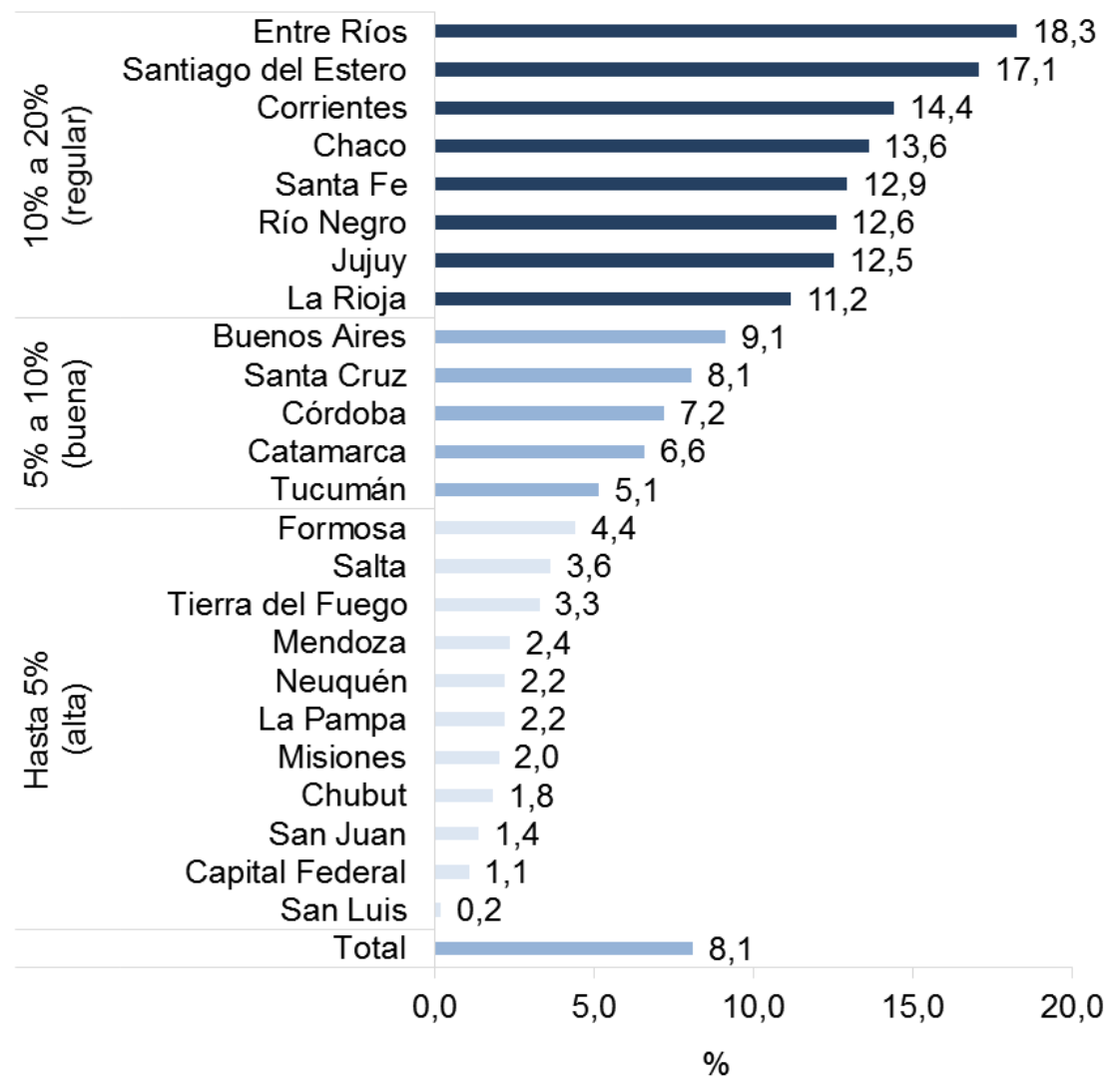

Elaboración propia con base a la cuadro 1 del Anexo.

Figura 1. PCMD en registros de defunción, Argentina según provincias y nivel de exactitud, 2011-2013.

Como se observa, la Argentina posee $8,1 \%$ de defunciones con causas mal definidas. En cuanto a la distribución provincial de dicho indicador se distingue una importante variabilidad (desviación estándar de 5,3 puntos porcentuales con respecto a la media), aunque ninguna jurisdicción se encuentra por encima de la categoría indicativa de exactitud baja ( $20 \%$ o más).

Los niveles de exactitud más altos se registran en 11 jurisdicciones, que muestran un PCMD de $1,8 \%$ en promedio. Este grupo incluye a San Luis, Capital Federal, San Juan, Chubut, Misiones, La Pampa, Neuquén, Mendoza, Tierra del Fuego, Salta y Formosa.

Otras cinco provincias argentinas poseen exactitud buena en la información sobre la causa básica de muerte, con un promedio de $8,5 \%$ de defunciones mal definidas. Dicho grupo está integrado por Tucumán, Catamarca, Córdoba, Santa Cruz y Buenos Aires. Esta última provincia -la más poblada del país-, se ubica por encima de la media nacional.

Finalmente, ocho provincias tienen un nivel regular de exactitud en los datos sobre la causa de muerte, con un PCMD medio de 14,1\%. Estas provincias son La Rioja, Jujuy, Río Negro, Santa Fe, Chaco, Corrientes, Santiago del Estero y Entre Ríos.

En la tabla 2 se presenta la distribución relativa de las causas mal definidas, según un agrupamiento básico de los códigos más frecuentes, entre los años 2011-2013. Como se distingue, las muertes se concentran fuertemente en el grupo de códigos correspondiente a las entidades mal definidas y desconocidas $(78,3 \%)$, seguidas por los signos y síntomas $(21,6 \%)$ y finalmente, por los hallazgos clínicos y de laboratorio $(0,03 \%)$. En el primer conjunto, los códigos más usuales corresponden a "Otras causas mal definidas y las no especificadas de mortalidad" (R99) y "Otras muertes súbitas de causa desconocida" (R96). 
Tabla 2. PCMD en registros de defunción, por tipo. Argentina según provincia, 2011-2013.

\begin{tabular}{|c|c|c|c|c|c|}
\hline Exactitud & Provincia & $\begin{array}{l}\text { Cantidad } \\
\text { (promedio) }\end{array}$ & $\begin{array}{l}\text { Síntomas y } \\
\text { Signos }\end{array}$ & $\begin{array}{l}\text { Hallazgos } \\
\text { anormales }\end{array}$ & Mal def. y desc. \\
\hline \multirow{11}{*}{$\begin{array}{l}\text { Hasta 5\% } \\
\text { (alta) }\end{array}$} & San Luis & 6 & 41,2 & 0,0 & 58,8 \\
\hline & Capital Federal & 342 & 35,6 & 0,0 & 64,4 \\
\hline & San Juan & 64 & 24,3 & 0,0 & 75,7 \\
\hline & Chubut & 55 & 36,9 & 0,0 & 63,1 \\
\hline & Misiones & 129 & 17,3 & 0,0 & 82,7 \\
\hline & La Pampa & 55 & 61,4 & 0,0 & 38,6 \\
\hline & Neuquén & 64 & 7,3 & 0,0 & 92,7 \\
\hline & Mendoza & 307 & 26,1 & 0,1 & 73,8 \\
\hline & Tierra del Fuego & 16 & 17,0 & 0,0 & 83,0 \\
\hline & Salta & 266 & 10,5 & 0,0 & 89,5 \\
\hline & Formosa & 151 & 0,4 & 0,0 & 99,6 \\
\hline \multirow{5}{*}{$\begin{array}{l}5 \% \text { a } 10 \% \\
\text { (buena) }\end{array}$} & Tucumán & 504 & 41,1 & 0,1 & 58,8 \\
\hline & Catamarca & 149 & 0,9 & 0,0 & 99,1 \\
\hline & Córdoba & 2.014 & 65,6 & 0,0 & 34,4 \\
\hline & Santa Cruz & 109 & 0,6 & 0,0 & 99,4 \\
\hline & Buenos Aires & 12.071 & 24,1 & 0,0 & 75,9 \\
\hline \multirow{8}{*}{$\begin{array}{l}10 \% \text { a } \\
20 \% \\
\text { (regular) }\end{array}$} & La Rioja & 230 & 14,3 & 0,0 & 85,7 \\
\hline & Jujuy & 517 & 3,9 & 0,0 & 96,1 \\
\hline & Río Negro & 505 & 16,3 & 0,1 & 83,6 \\
\hline & Santa Fe & 3.773 & 8,1 & 0,0 & 91,9 \\
\hline & Chaco & 965 & 6,7 & 0,0 & 93,3 \\
\hline & Corrientes & 963 & 6,7 & 0,0 & 93,3 \\
\hline & $\begin{array}{l}\text { Santiago del } \\
\text { Estero }\end{array}$ & 914 & 6,2 & 0,2 & 93,6 \\
\hline & Entre Ríos & 1.814 & 12,7 & 0,0 & 87,3 \\
\hline Total & & 25.983 & 21,6 & 0,0 & 78,3 \\
\hline
\end{tabular}

Procesamientos especiales de bases de datos de la DEIS, Argentina.

En el segundo, los códigos más utilizados son "Choque cardiogénico" (R57.0), "Paro respiratorio" (R09.2) y "Senilidad" (R54). Por último, en el tercer grupo de códigos se destaca "Hiperglicemia, no especificada" (R73.9), siendo de todas formas muy poco habitual.

La distribución de los códigos es muy variable en las provincias (tabla 1). Sin embargo, se reconoce que las causas imprecisas y desconocidas se concentran menos en los grupos de jurisdicciones de exactitud alta y buena (70,1\% y $77,1 \%$ respectivamente), que en las provincias de precisión regular $(91,1 \%)$. Lo contrario se observa en cuanto a los códigos que se refieren a síntomas y signos.

Como se expone en la Figura 2, el país incrementa en 12,9\% el PCMD entre los trienios 2001-03 y 2011-13. En las provincias se observan dos pautas diferentes. Las jurisdicciones con los niveles más elevados de exactitud en la información sobre la causa básica de muerte, presentan descensos en el PCMD entre 2001-2003 y 2011-2013. Salvo muy pocas excepciones, las provincias con niveles buenos y regulares de precisión en la asignación de la causa de muerte, registran la tendencia contraria entre los mismos trienios. 


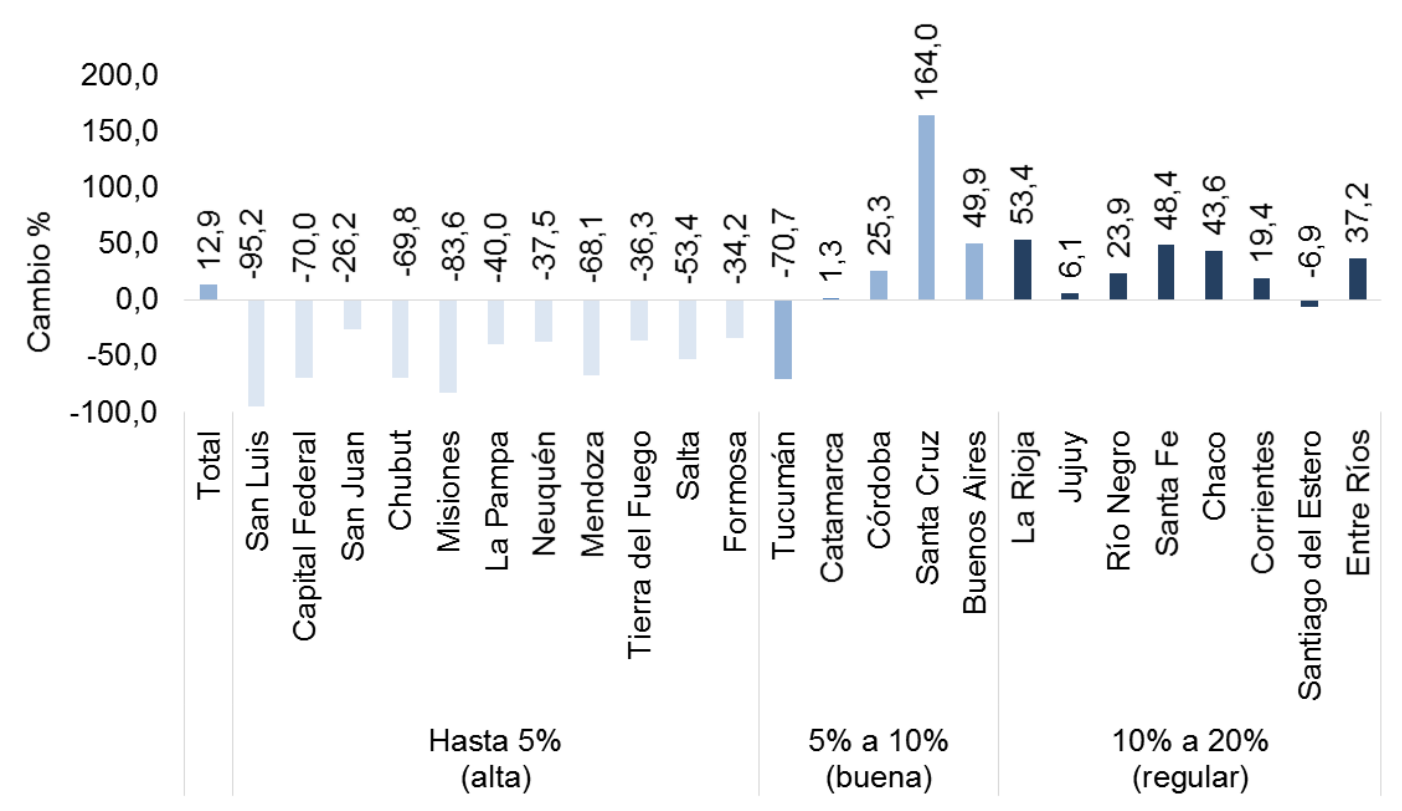

Elaboración propia con base a la cuadro 1 del Anexo.

Figura 2. Cambio porcentual de PCMD en registros de defunción entre los años 2001-2003 y 2011-2013. Argentina según provincias.

En el primer grupo de provincias (exactitud excelente), se destacan especialmente las reducciones absolutas observadas en la Ciudad Autónoma de Buenos Aires y Mendoza (de 2.700 a 1.700 defunciones mal definidas menos, aproximadamente), y los cambios porcentajes de San Luis $(-95,2 \%)$ y Misiones $(-83,6 \%)$.

Entre las provincias del segundo grupo (precisión buena), sólo se registra un descenso en Tucumán $(-70,7 \%)$. Este a su vez corresponde a la disminución absoluta más importante ocurrida en el país entre 2001-2003 y 2011-2013 (3.100 defunciones con causas mal definidas). El resto de las jurisdicciones que componen este grupo exhibe incrementos más o menos relevantes en las causas mal definidas. En términos absolutos, la provincia que más defunciones aporta en estas condiciones es Buenos Aires (14.000 muertes con causas mal definidas). En porcentajes, el aumento más notable corresponde a Santa Cruz (164\%).

Finalmente, en el tercer grupo casi todas las provincias presentan aumentos en las defunciones con causas mal definidas. La provincia con mayor incremento absoluto de este problema corresponde a Santa $\mathrm{Fe}$ (4.100 las defunciones con causas definidas incorrectamente). En cuanto al cambio porcentual, la provincia que más aumento registra es La Rioja $(53,4 \%)$. En este conjunto, sólo Santiago del Estero reduce el PCMD entre los periodos en estudio $(-6,9 \%)$.

\section{DISCUSIÓN}

El sistema de información sobre mortalidad de la Argentina cuenta con una larga trayectoria. En cuanto a la producción de datos, los esfuerzos realizados han redundado en una cobertura del registro prácticamente completa (15-17), y en la difusión oportuna y diversificada de los resultados en bases de datos, publicaciones impresas y digitales (18). Asimismo, desde hace tiempo se realizan esfuerzos para mejorar la exactitud de la información sobre defunciones. Algunos ejemplos son la modificación del cuestionario utilizado para recolectar los datos, aprobado en 1999 e implementado en el año 2001, y el diseño de cursos y manuales de apoyo, dirigidos a los agentes encargados de completarlo y codificarlo (18-20).

En cuanto a la exactitud de la información sobre la causa básica de muerte, y en concordancia con otros estudios de alcance nacional o regional $(3,11,13)$, en este trabajo se ha mostrado que Argentina presenta un nivel aceptable de causas mal definidas $(8,1 \%$ del total de defunciones entre 2011-2013). No obstante, debe tenerse presente que la cifra actual representa un crecimiento de casi $13 \%$ respecto a 2001-2003, y que de continuar con esta tendencia el análisis de los datos sobre la causa de muerte podría verse comprometido en términos de exactitud. 
Asimismo, esta investigación muestra que el promedio argentino del PCMD, involucra situaciones provinciales diferentes. Según se constata, 11 jurisdicciones presentan un nivel excelente de exactitud, 5 un nivel bueno y 8 un nivel regular. En este último conjunto, debe prestarse especial atención a las provincias de Santiago del Estero y Entre Ríos, cuyo nivel de mala definición es próximo al $20 \%$. La tendencia temporal en la mala definición de las causas de muerte, también se manifiesta diferencialmente a nivel provincial. Existen reducciones en el PCMD de todas las jurisdicciones que pertenecen al primer grupo (exactitud alta), mientras que lo mismo ocurre en sólo dos del segundo y tercer grupo (exactitud buena o regular respectivamente) ${ }^{1}$.

Otro examen de interés, se refiere al tipo de códigos más frecuentes en la mala definición de la causa de muerte. En Argentina, la gran mayoría corresponde a causas desconocidas o mal definidas propiamente dichas, seguidas escasamente por los signos y síntomas, y aún menos, por los hallazgos anormales clínicos y de laboratorio. En cada uno de estos conjuntos, son muy pocos los códigos puntualmente utilizados (por ejemplo; R99, R96, R57.0 y R09.2). A grandes rasgos, en las provincias argentinas se presenta la misma pauta de "repetición" de los códigos en la mala definición. La excepción está dada en que el grupo de causas más habituales (desconocidas o mal definidas propiamente dichas), suele ser proporcionalmente menos relevante en las jurisdicciones que poseen un nivel excelente de exactitud.

El mejoramiento de la información sobre la causa básica de muerte, constituye una necesidad para la planificación pública que la utiliza para la definición y seguimiento de políticas y programas de salud, y por ende, para la comunidad receptora de los mismos. Con respecto a los hallazgos de esta investigación, algunas recomendaciones preliminares se relacionan con la posibilidad de reconocer y difundir las buenas prácticas de las provincias que han logrado reducir el problema estudiado, y de implementar programas focalizados en las jurisdicciones más afectadas.

También puede tenerse en cuenta la experiencia de otros países de América Latina, entre los que se han encontrado diferenciales político-administrativos similares en la exactitud de la causa básica de muerte. Por ejemplo, entre los servicios de salud de Chile (21), departamentos de Colombia (22), estados de Brasil (23) y regiones de Cuba (24). La idea se corresponde con la propuesta general de la Red Latinoamericana y del Caribe para el Fortalecimiento de los Sistemas de Información en Salud (RELACSIS, OPS/OMS), que difunde y promueve experiencias exitosas en los países de la región.

La bibliografía internacional indica, asimismo, que el mejoramiento de estas estadísticas requiere la capacitación y entrenamiento de los médicos, tanto en el llenado de las formas como en los principios de la CIE-10 $(6,25)$. En Argentina, la DEIS ha diseñado un curso de excelente calidad, pero el mismo puede ofrecerse sólo de manera optativa, a partir de una plataforma web (20). En este aspecto, podría reforzarse el rol de las universidades y los colegios profesionales de medicina, agregando oficialmente en los planes de estudio la formación y el entrenamiento en la certificación de la causa de muerte.

A propósito de las prácticas de certificación mencionadas, también es importante recordar que la intervención del médico debería realizarse en el marco adecuado. Aunque no existen evidencias concretas en Argentina, es posible que ante el exceso de actividades y de responsabilidades administrativas los profesionales deleguen la tarea a practicantes $u$ otros agentes médicos o administrativos. Además, es sabido que muchos médicos perciben la ausencia de garantías legales para el reporte de ciertas causas de muerte; especialmente, de aquellas relacionadas con hechos violentos (6).

Es importante tener presente las limitaciones de esta investigación. En tanto constituye una primera aproximación a la evaluación de las estadísticas sobre mortalidad de las provincias de Argentina, el análisis debería ser complementado por otros enfoques tanto directos como indirectos. En primer término, el enfoque metodológico que se utiliza no reflejaría la magnitud y tipología de todos los errores de exactitud que pueden afectar a las causas de muerte. Por ello, y en términos de evaluación indirecta, en las provincias de Argentina también puede tenerse presente el análisis de los llamados códigos "basura" $(26,27)$, causas "imprecisas" (28) o "poco útiles" de defunción (29). Además de las causas mal definidas, este enfoque también identifica otros casos de certificación incompleta o incorrecta de la causa básica de muerte que sería de utilidad identificar; por ejemplo, el uso

\footnotetext{
1 - Los informes técnicos existentes sobre las estadísticas vitales de la Ciudad Autónoma de Buenos Aires, coinciden en indicar el alto nivel de exactitud de la causa de muerte $(5,30)$. En el último de estos informes también se destaca un descenso de la mala definición entre los años 2004 y 2008.
} 
de formas de morir como causas básicas o síntomas de éstas (causas inmediatas e intermedias respectivamente).

Asimismo, es relevante señalar que la evaluación de esta fuente de datos se enriquecería considerablemente mediante estudios ulteriores basados en metodologías directas, como la autopsia verbal. La técnica permite reconstruir las causas de muerte a través de entrevistas a los agentes médicos, no médicos y familiares que han sido testigos de la defunción, con lo cual se obtiene un parámetro válido para comparar con la certificación oficialmente realizada.

\section{REFERENCIAS BIBLIOGRÁFICAS}

1. World Health Organization. International statistical classification of diseases and related health problems. - 10th revision, edition 2010 [Internet]. Malta: WHO; 2011. 195 p. Disponible en: http://www.who.int/classifications/icd/ICD10 Volume2_en_2010.pdf?ua $=1$

2. Naciones Unidas. Departamento de Asuntos Económicos y Sociales. División de Estadística. Principios y recomendaciones para un sistema de estadísticas vitales. Revisión 2. Nueva York: NNUU; 2003.

3. Ribotta BS, Escanés G. Códigos "poco útiles" en los registros de defunción en Argentina, Chile, Colombia y México (2000-2011). Med Salud y Soc. En prensa; 2014;5(1):4-17.

4. Organización Mundial de la Salud. Estadísticas Sanitarias Mundiales 2014. Organización Mundial de la Salud, editor. Ginebra; 2014. 178 p.

5. Mazzeo V. ¿Qué debemos mejorar en el registro de las estadísticas vitales ? Población de Buenos Aires [Internet]. 2005;2(2):69-78. Disponible en: http://www.redalyc.org/pdf/740/74020209.p df

6. World Health Organization. Strengthening civil registration and vital statistics for births, deaths and causes of death: resource kit. HMN/WHO/The University of Queensland/AustralianAID, editor. Luxembourg: WHO; 2013.

7. Organización Panamericana de la Salud. Clasificación estadística internacional de enfermedades y problemas relacionados con la salud - Décima revisión. Volumen 1: Lista tabular. [Internet]. 2005th ed. OPS/OMS, editor. Washington, D.C.: OPS/OMS; 1995. Disponible

en: http://ais.paho.org/classifications/Chapters/ pdf/Volume1.pdf

8. Abouzahr C, Mikkelsen L, Rampatige R, Lopez A. Mortality statistics: a tool to improve understanding and quality [Internet]. Herston; 2010. Report No.: 13. Disponible en: http://www.uq.edu.au/hishub/docs/WP13/HI SHub-WP13-11-WEB 2Oct12.pdf

9. Organización Mundial de la Salud. Estadísticas Sanitarias Mundiales 2012 [Internet]. Ginebra: Organización Mundial de la Salud; 2012. 178 p. Disponible en: http://apps.who.int/iris/bitstream/10665/44 858/1/9789243564449_spa.pdf

10. Mathers CD, Fat DM, Inoue M, Rao C, Lopez $A D$. Counting the dead and what they died from: an assessment of the global status of cause of death data. Bull World Health Organ [Internet]. 2005;83(3):171-7. Disponible en: http://www.pubmedcentral.nih.gov/articlere nder.fcgi?artid=2624200\&tool=pmcentrez\&r endertype $=$ abstract

11. Gawryszewski V, Sanhueza A, Solíz P, Giusti A. Trends in ill-defined causes of deaths in Latin America, 2000 - around 2010. En: RELACSIS, editor. $V$ Reunión RELACSIS. México: PAHO; 2013.

12. Argentina. Ministerio de Salud. Dirección de Estadísticas e Información en Salud. Conclusiones y recomendaciones. XII Reunión Nacional de Estadísticas de Salud; Buenos Aires 201031 agosto - 1 de setiembre [Internet]. Buenos Aires: Ministerio de Salud de la Nación; 2010. p. 116. Disponible en: http://www.deis.gov.ar/publicaciones/Archiv os/Serie3Nro54.pdf

13. Ribotta BS. Precisión de los datos sobre defunciones del Sub-Sistema de Estadísticas Vitales de la Argentina (2001-2009). En: AEPA, editor. XII Jornadas Argentinas de Estudios de Población. Bahía Blanca: AEPA; 2013. p. 18-20.

14. Marconi E, Guevel C, Fernández M. Estadísticas Vitales. En: Torrado S, editor. Población y bienestar en la Argentina del primero al segundo Centenario Una historia social del siglo XX. Buenos Aires: Edhasa; 2007. p. 287-308.

15. Organización Panamericana de la Salud. Argentina. Situación de las estadísticas vitales y de salud del país hacia 2005. Informe de la estrategia para el fortalecimiento de las estadísticas vitales y de salud de los países de las Américas. Washington DC; 2007.

16. Bay G, Orellana H. La calidad de las estadísticas vitales en la América Latina. Taller de expertos en el uso de estadísticas vitales: alcances y limitaciones; Santiago de Chile 2007 13-14 de diciembre. Santiago [Chile]: CELADE/CEPAL; 2007.

17. World Health Organization. Demographic and socioeconomic statistics. Census and civil registration coverage by country: global health observatory data repository [Internet]. 2014. p. 1-2. Disponible en: http://apps.who.int/gho/data/node.main. 12 1 ?lang $=$ en

18. Argentina. Ministerio de Salud. Dirección de Estadísticas e Información en Salud. Sistema 
de información de salud: edición actualizada - diciembre 2004. Buenos Aires: MINSAL; 2005.

19. Argentina. Ministerio de Salud. Dirección de Estadísticas e Información en Salud. Modelos de formularios e instructivos del sistema de estadísticas sociales. Buenos Aires: MINSAL; 2000.

20. Argentina. Ministerio de Salud. Dirección de Estadísticas e Información en Salud. La participacion del médico en las estadísticas de mortalidad [Internet]. 2006. p. 2. Disponible en: http://www.deis.gov.ar/Capacitacion/home. htm

21. Núñez L, Icaza G. Calidad de las estadísticas de mortalidad en Chile, 1997-2003. Rev Med Chil. 2006;(134):1191-6.

22. Cendales R, Pardo C. La calidad de certificación de la mortalidad en Colombia, 2002-2006. Rev Salud Pública [Internet]. 2011 [cited 2014 Feb 16];13(2):229-38. Disponible en: http://www.scielosp.org/pdf/rsap/v13n2/v13 n2a05.pdf

23. Paes NA. Qualidade das estatísticas de óbitos por causas desconhecidas dos Estados brasileiros. Rev Saúde Pública. 2007;41(3):436-45.

24. Gran-Álvarez $M$, Zacca Peña $E$, Fernández Viera M, Martinez Morales M. Calidad de las estadísticas de mortalidad en provincias cubanas según cuantificación de causas de muerte imprecisas. Rev Cuba Salud Pública [Internet]. 2010;36(2):109-15. Disponible en:

http://scielo.sld.cu/scielo.php?pid=S086434662010000200003\&script $=$ sci_arttext\&tln $\mathrm{g}=\mathrm{pt}$

25. Mahapatra P, Shibuya K, Lopez AD, Coullare
F, Notzon FC, Rao C, et al. Civil registration systems and vital statistics: successes and missed opportunities. Lancet [Internet]. 2007;370(9599):1653-63. Disponible en: http://www.ncbi.nlm.nih.gov/pubmed/18029 006

26. Murray C, Lopez AD. The global burden of diseases. A comprehensive assessment of mortality and disability from diseases, injuries and risk factors in 1990 and projected to 2020 [Internet]. Harvard School of Public Health. WHO. World Bank, editor. Washington DC: Harvard University Press; 1996. 940 p. Disponible en: https://extranet.who.int/iris/restricted/bitstr eam/10665/41864/1/0965546608_eng.pdf

27. Naghavi M, Makela S, Foreman K, O'Brien J, Pourmalek F, Lozano R. Algorithms for enhancing public health utility of national causes-of-death data. Popul Health Metr [Internet]. 2010;8(9):1-14. Disponible en: http://www.pubmedcentral.nih.gov/articlere nder.fcgi?artid=2873308\&tool=pmcentrez\&r endertype $=$ abstract

28. Zacca Peña $E$, Gran Álvarez M, Martinez Morales M, Fernandez Viera M. Calidad de las estadísticas de mortalidad en Cuba según cuantificación de causas de muerte imprecisas. Rev Cuba Salud Pública. 2010;36(2):102-8.

29. OPS/OMS. Causas de defunción poco útiles [Internet]. Washington. 2014. p. 1. Disponible en: http://www.paho.org/hq/index.php?option= com_content\&view $=$ article $\& i d=6788 \&$ catid $=$ 2390: themes\&Itemid $=2391$ \&lang $=$ es

30. Govea Basch J. Lo que todavía debemos mejorar en el registro de las estadísticas vitales. Población de Buenos Aires. 2010;7(11):63-74. 